\title{
Concentration Micro-field for Lamellar Eutectic Growth
}

\author{
Waldemar Wołczyński \\ Institute of Metallurgy and Materials Science, \\ Polish Academy of Sciences, \\ 30059 Kraków, Reymonta 25, Poland \\ email: nmwolczy@imim-pan.krakow.pl
}

\begin{abstract}
Keywords: diffusion equation, oriented growth, solute concentration, phase protrusion, mass
\end{abstract} balance, coupled growth, liquid phase separation.

\begin{abstract}
A possibility of a modification of the Jackson-Hunt theory of an oriented structure formation is analysed. A new model for the formation of a concentration field ahead of growing regular lamellae with respect to the solid / liquid interface shape is presented. A coordinate system applied in the model is attached to the solid / liquid interface to be advancing in the $z$ - direction, identically with interface moving at a constant velocity, $v$. The solution to a diffusion equation is given for the improved formulation of the boundary conditions. The boundary conditions are related to the interplay between the diffusion required for phase separation and the formation of the interphase between both lamellae. The boundary conditions are formulated to establish the stability of lamellar structure formation under steady-state conditions. It is assumed that stable growth of the lamellae is ensured by the separation of concentration fields within a boundary layer ahead of the solid / liquid interfaces of both the $\alpha$ and $\beta$. phases. Coupled lamellar growth with the presence of a leading phase protrusion is defined. The general mass balance is analysed for a solute concentration in the liquid, taking into account a planar solid / liquid interface. A local mass balance is also ensured but it requires envisaging a protrusion of the minor eutectic phase. The existence of a lead distance is confirmed experimentally for the $(\mathrm{Pb})-(\mathrm{Cd})$ eutectic system. The difference in undercooling is also considered as a phenomenon associated with the separation of concentration fields and the existence of a protrusion to relax the assumption of an isothermal interface (ideally coupled growth) given by the Hunt and Jackson theory.
\end{abstract}

\section{Analysis of the Jackson-Hunt theory}

The foundations for a theoretical description of mass transport ahead of a solid / liquid interface of growing eutectics presenting lamellar structure has been laid by Jackson-Hunt [1]. The solution of the diffusion equation (known as the J-H theory):

$$
\nabla^{2} C+\frac{v}{D} \frac{\partial C}{\partial z}=0, \quad \text { with } \quad C=C_{E}+C_{\infty} \quad \text { at } \quad z=\infty
$$

was found by Jackson-Hunt [1] under the assumption that the solid / liquid interface is planar. The $\mathrm{J}-\mathrm{H}$ theory, that is, a steady-state solution to the diffusion equation, Eq. (1), for lamellar eutectic growth at a constant growth rate, $v$, with a planar $s / 1$ interface was given with liquid on or off eutectic composition. Thus,

$$
\frac{\partial C}{\partial x}=0, \quad \text { at } \quad x=0 \quad \text { and } \quad x=S_{\alpha}+S_{\beta}
$$

in the $\mathrm{J}-\mathrm{H}$ theory. The conservation of the mass balance suggested by the $\mathrm{J}-\mathrm{H}$ theory is given by: 


$$
\frac{\partial C}{\partial z}=\mp \frac{v C_{0}^{S}}{D} ; \quad(S=\alpha, \beta) \quad \text { at } \quad z=0, \quad \text { for } \quad 0 \leq x<S_{\alpha} \quad \text { and } \quad S_{\alpha}<x \leq S_{\alpha}+S_{\beta} .
$$

The solution of Eq. (1) given by the J-H theory is plotted schematically in Fig. 1a with the corresponding undercooling (Fig. 1b) and interface curvature (Fig. 1c) [2].
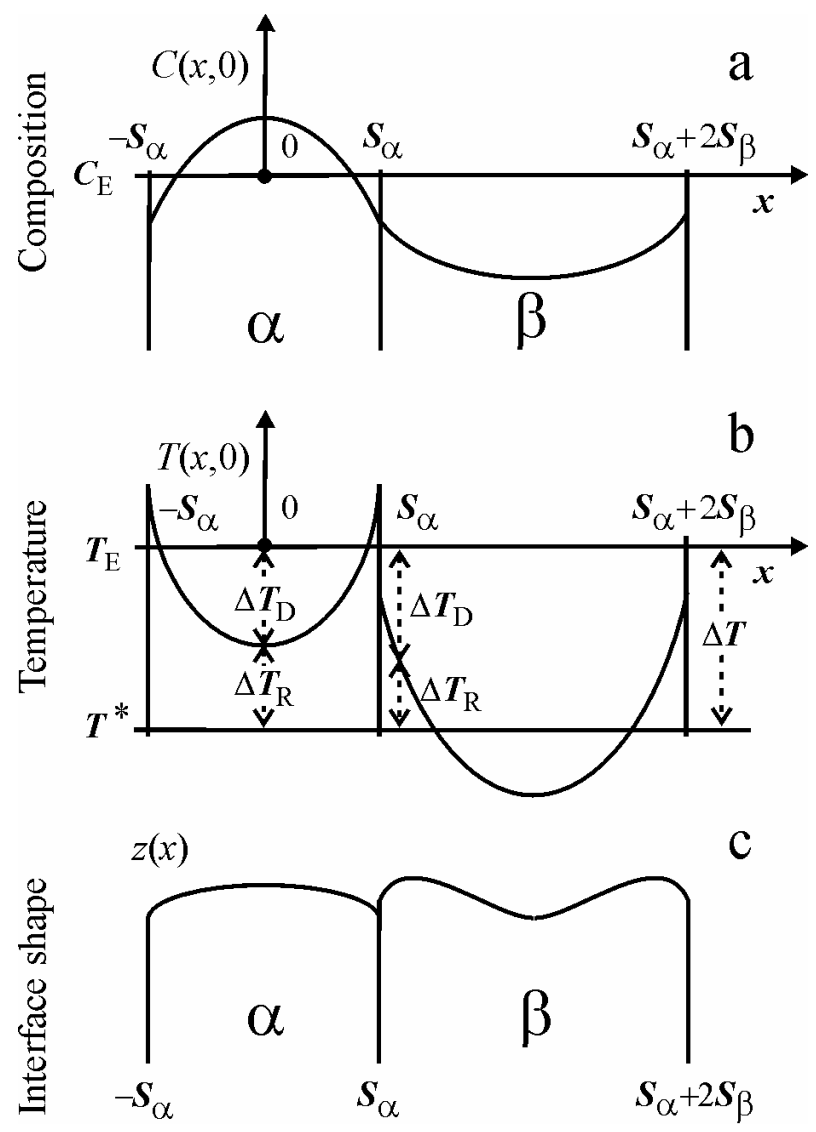

Fig. 1 Scheme of the solid / liquid interface showing a plot of: a.) interface composition, b.) solute undercooling, $\Delta T_{D}$, and curvature undercooling $\Delta T_{R}$, c.) interface shape [2].

According to the scheme shown in Fig. 1a some parts of the $\alpha$ - phase should grow from the liquid of a solute content adequate for the formation of the $\beta$ - phase. This could lead to changes in the inter-lamellar spacing. Some changes of the spacing are possible during the real solidification as described in the Magnin-Trivedi model, where adjustments of the inter-lamellar spacing during composite growth are explained from the viewpoint of the behaviour of a mechanical equilibrium existing at the triple point of the solid / liquid interface [3].

An instability of a lamellar structure formation is also the subject of current analysis (Fig. 2a). The observation is compared with some theoretical predictions given in the J-H theory (Fig. 2b). The J-H stability / instability considerations are developed in several treatments dealing with the s/1 interface response to an imposed perturbation [4-8].
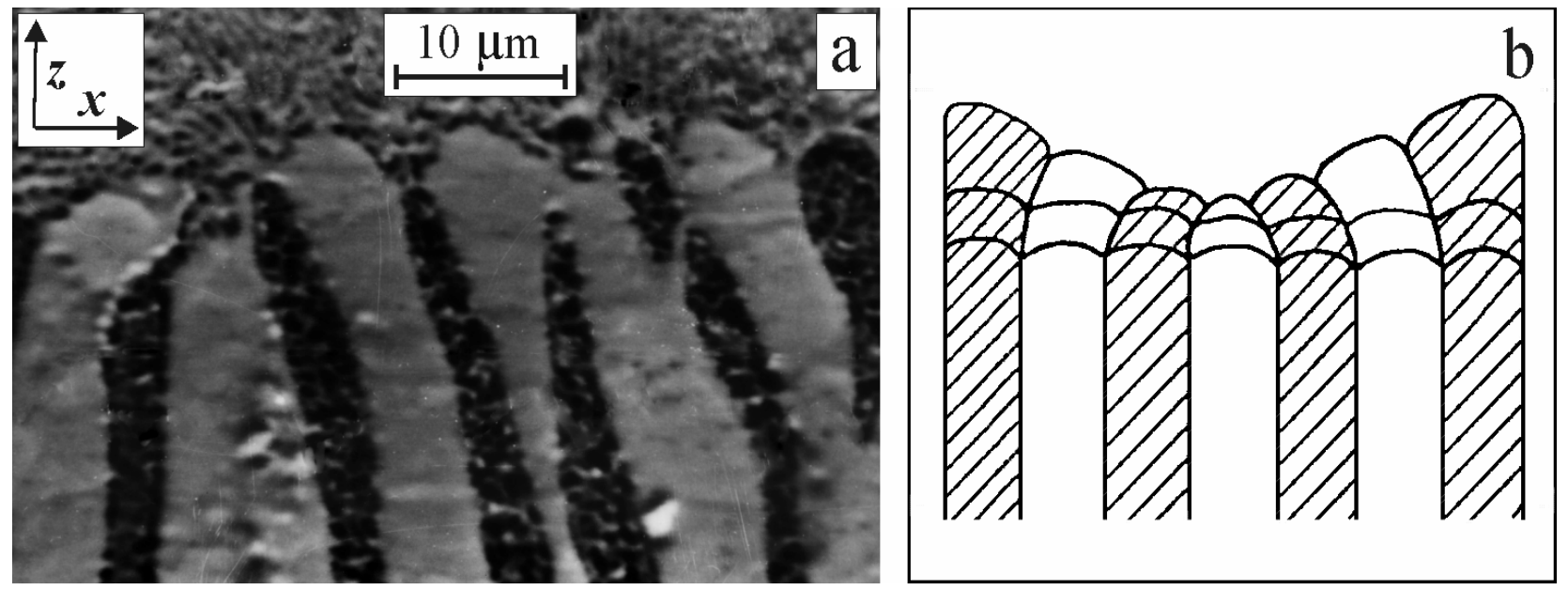

Fig. 2 Instability of the $\mathrm{s} / \mathrm{l}$ interface of a composite in situ: a.) observed during the real solidification, b.) concluded due to the $\mathrm{J}-\mathrm{H}$ theory. 
Detailed analysis of the J-H theory leads to the conclusion that the volumes of two solid phases of a composite should be equal to each other to ensure the stable growth as it results from the scheme illustrated in Fig. 1a. That is why, it is evident that the J-H theory is an excellent approximation only for the symmetrical eutectic phase diagrams. However, in reality there are no symmetrical diagrams and this restriction should be removed from the theory of formation of the concentration micro-field created ahead of the s/l interface.

The boundary conditions applied to the current analysis ensure not only an improved description of the concentration micro-field for the formation of an eutectic structure of an alloy from real asymmetrical phase diagrams but also a separation of the liquid from which the different solid phases are growing. Contrary to the J-H theory, the improved concentration micro-field should be favourable for the correct description of a stable formation of a directional eutectic structure.

The assumption of non separation of concentration fields in the liquid applied in the J-H theory together with the concept of ideally coupled growth $\left(\Delta T_{\alpha}^{*}=\Delta T_{\beta}^{*}=\Delta T\right.$, Fig. 1b), results in a discontinuity of solute undercooling as well as temperature at the $\alpha / \beta$ - inter-phase, Fig. $1 \mathrm{~b}$. According to the scheme shown in Fig. 1b, some parts of the $\alpha$ - phase lamella should grow outside the assumed regime of the ideally coupled growth: $T^{*}-T_{E}$. Analogously, the middle part of the $\beta$ - phase lamella should also grow outside the mentioned regime: $T^{*}-T_{E}$, Fig. $1 \mathrm{~b}$. Moreover, the curvature undercooling compensating the behaviour of the solute undercooling within the regime of ideally coupled growth: $T^{*}-T_{E}$ produces a non realistic shape of the $\mathrm{s} / 1$ interface, Fig. 1c. A detailed analysis of the scheme shown in Fig. 1a [2], indicates that the concentration profile is not correct. This type of profile can be observed when the $\alpha$ - phase is represented by wider lamella than the $\beta$ - phase, as it results from the solution of Eq. (1) for which a mass balance is satisfied at each $z$ - coordinate. However, the fulfilment of the mass balance is not visible in Fig. 1a.

The corrected scheme for the solution of Eq. (1) is shown in Fig. 3a. This corresponds to the phase diagram (see Fig. 4b) according to which $\left|C_{0}^{\alpha}\right|<\left|C_{0}^{\beta}\right|$ and in consequence $S_{\alpha}>S_{\beta}$ as is observable in Fig. 4a. The condition mentioned above is not fulfilled in Fig. 1a plotted by HuntJackson [2], and reproduced further by Elliott [9], Rutter [10], and Kurz-Fisher [11].

Fig. 3 Corrected schemes of: a.) solute profile with satisfaction of mass balance at $z=0$ and $\mathrm{b}$.) adequate solute and curvature undercooling of the solid/liquid interface according to the $\mathrm{J}-\mathrm{H}$ theory.

The solute undercooling corresponding to the proper concentration profile for the J-H theory is shown schematically in Fig. $3 b$. The concentration micro-field shown in Fig. 1a as well as the undercooling, Fig. 1b could be illustrated on the phase diagram. This illustration is plotted schematically in Fig. 5.
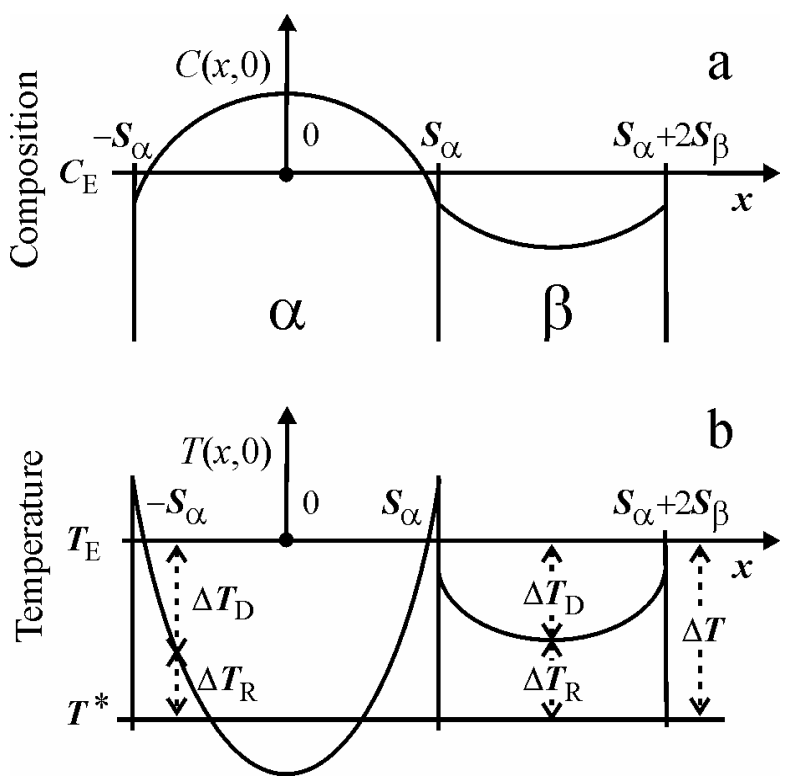

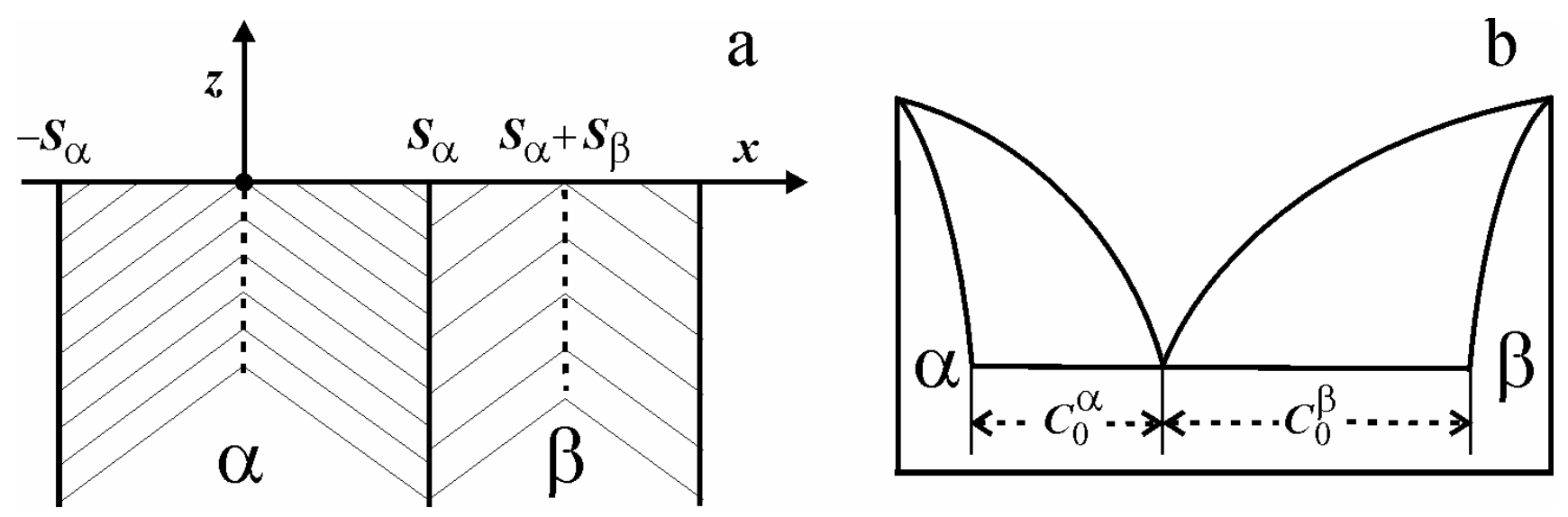

Fig. 4 Schemes of a.) planar lamellar composite in situ interface showing geometrical definitions of the structure, b.) phase diagram giving the definitions of both parameters $C_{0}^{\alpha}$ and $C_{0}^{\beta}$, related to the width of both lamellae.

Fig. 5 Interpretation of the concentration micro-field and undercooling both given in Fig. 1.

However, taking into account that the scheme shown in Fig. 1 was corrected in Fig. 3 an adequate interpretation should also be improved. A phase diagram with the corrected concentration field and undercooling is given in Fig. 6.

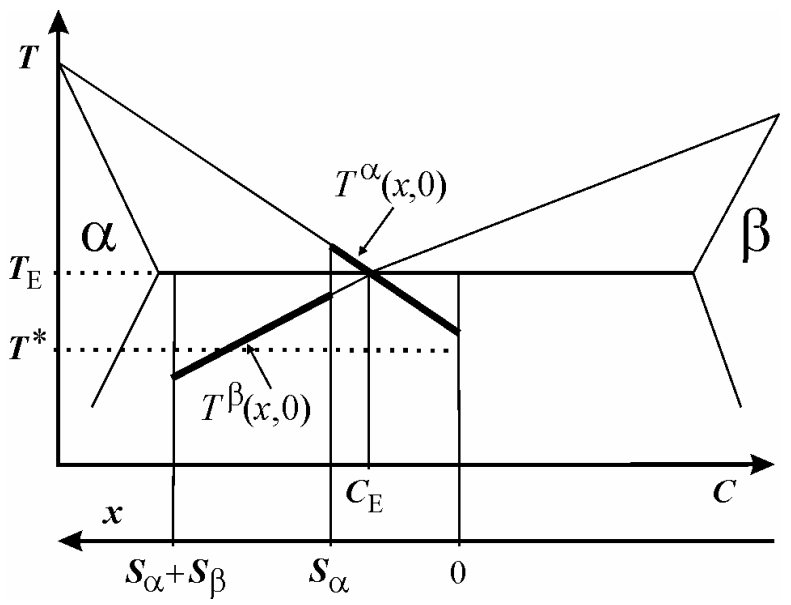

Fig. 6 Interpretation of the corrected concentration micro-field and undercooling from Fig. 3.

The boundary condition, Eq. (3) applied in the $\mathrm{J}-\mathrm{H}$ theory contains the constant $C_{0}^{S}$, ( $S=\alpha, \beta)$, defined in Fig. $4 \mathrm{~b}$, instead of the unknown function $C(x, 0)$. This restriction could be eliminated from the theory of in situ growth of composites but it requires an application of a numerical treatment of the solution to the diffusion equation, Eq. 1.

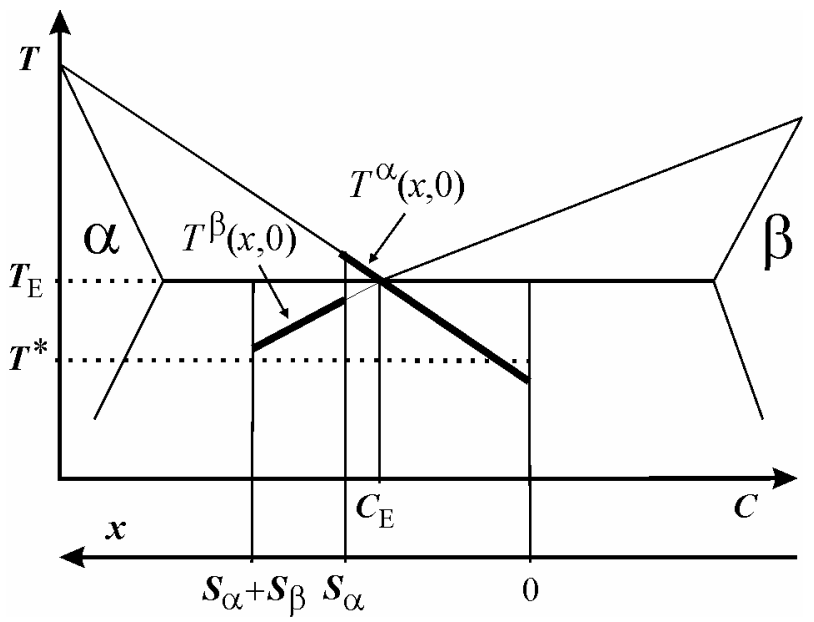
An analytical solution with the adaptation of the $C(x, 0)$-function does not exist in the literature. 


\section{Fundamentals of the current model}

The foundations for the current analysis of the concentration field ahead of the solid/liquid interface of an in situ composite of lamellar structure are improved in comparison with those of the $\mathrm{J}-\mathrm{H}$ theory. First of all, the current model is no longer associated with the concept of an ideally coupled growth of an eutectic structure, that is, with $\Delta T_{\alpha}^{*}=\Delta T_{\beta}^{*}=\Delta T=\Delta T_{D}(x, 0)+\Delta T_{R}(x, 0)$.

The assumed separation of the concentration fields ahead of each lamella allows one to consider real asymmetrical phase diagrams. Moreover, an improved concentration field is adequate for the description of a stable formation of a directional eutectic structure. Therefore, the $\alpha$ - phase and the $\beta$ - phase lamellae are formed properly to their liquidus line positions within the zone of undercooling as shown in Fig. 7.

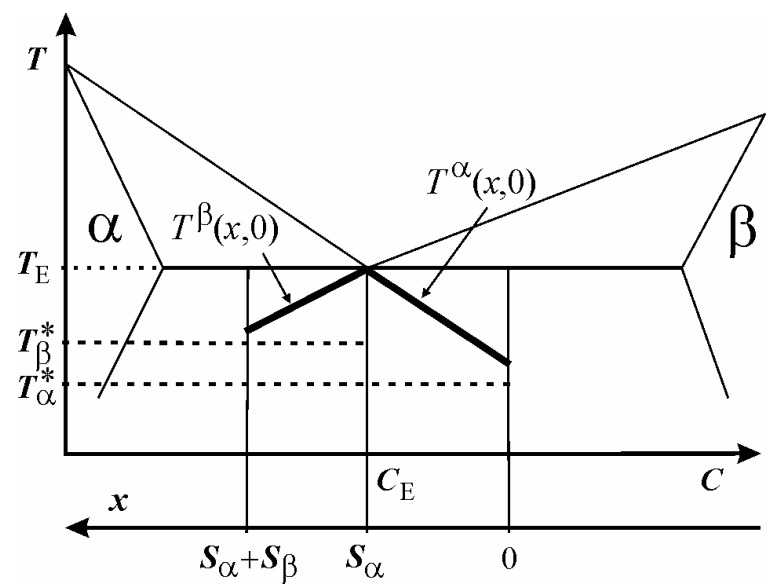

Fig. 7 Interpretation of the concentration micro-field and undercooling due to the current model.

According to the scheme plotted in Fig. 7 the following definitions are assumed in the proposed analysis associated with the concept of coupled growth $\left(\Delta T_{\alpha}^{*} \neq \Delta T_{\beta}^{*}\right)$ :

a/ for solute undercooling

$$
\delta T^{\alpha}(x, 0)=T^{\alpha}(x, 0)-T_{E}, \quad \text { and } \quad \delta T^{\beta}(x, 0)=T^{\beta}(x, 0)-T_{E},
$$

$\mathrm{b} /$ for curvature undercooling

$$
\delta T_{R}^{\alpha}(x, 0)=T_{\alpha}^{*}-T^{\alpha}(x, 0), \quad \text { and } \quad \delta T_{R}^{\beta}(x, 0)=T_{\beta}^{*}-T^{\beta}(x, 0),
$$

c/ for total undercooling, according to the concept of coupled growth

$$
\delta T^{\alpha}(x, 0)+\delta T_{R}^{\alpha}(x, 0)=\Delta T_{\alpha}^{*}, \quad \text { and } \quad \delta T^{\beta}(x, 0)+\delta T_{D}^{\beta}(x, 0)=\Delta T_{\beta}^{*},
$$

$\mathrm{d} /$ for the concentration micro-field

$$
\delta C^{\alpha}(x, 0)=C^{\alpha}(x, 0)-C_{E}, \quad \text { and } \quad \delta C^{\beta}(x, 0)=C^{\beta}(x, 0)-C_{E} .
$$

Consequently,

$$
C_{0}^{\alpha}\left(S_{\alpha}, 0\right)=C_{S}^{\alpha}\left(S_{\alpha}, 0\right)-C_{E}<0, \quad \text { and } \quad C_{0}^{\beta}\left(S_{\alpha}, 0\right)=C_{S}^{\beta}\left(S_{\alpha}, 0\right)-C_{E}>0 .
$$

Equation (8) describes parameters $C_{0}^{\alpha}$ and $C_{0}^{\beta}$ known in the J-H theory and shown in Fig. $4 \mathrm{~b}$. 
The boundary condition applied in the model is as follows (see Fig. 7):

$\delta C\left(S_{\alpha}, z\right)=C\left(S_{\alpha}, z\right)-C_{E}=0$.

The general solution of the diffusion equation:

$$
\frac{\partial^{2} \delta C}{\partial x^{2}}+\frac{\partial^{2} \delta C}{\partial z^{2}}+\frac{v}{D} \frac{\partial \delta C}{\partial z}=0
$$

is:

$$
\begin{aligned}
& \delta C(x, z)=X(x) Z(z), \\
& X(x)=A \cos (\omega x)+B \sin (\omega x), \quad Z(z)=\exp \left[\left(-\frac{v}{2 D}-\sqrt{\frac{v^{2}}{4 D^{2}}+\omega^{2}}\right) z\right]
\end{aligned}
$$

and: $A, B, \omega$ are to be defined.

The solutions are given separately for each of both lamellae under the assumption that the s/1 interface is planar, Fig. 4a. Thus,

A/ for the $\alpha$-phase lamella, that is, for $x \in\left[0, S_{\alpha}\right], z \geq 0$.

The values of the $B$ and $\omega$ parameters come from the conditions:

$$
\left.\frac{\partial \delta C(x, z)}{\partial x}\right|_{x=0}=0
$$

$\delta C\left(S_{\alpha}, z\right)=0$

It is evident from Eq. (13) that $-\omega A \sin (\omega \cdot 0)+\omega B \cos (\omega \cdot 0)=0$, and finally $B=0$ and from Eq. (13) and Eq. (14): $A \cos \left(\omega S_{\alpha}\right)=0$ and $\omega=\omega_{2 n-1}=\frac{(2 n-1) \pi}{2 S_{\alpha}}, \quad n=1,2, \ldots$.

Considering Eq. (11) and Eq. (12) with both of the above definitions a general solution of Eq. (10) with conditions given by Eq. (13) and Eq. (14) can be written as:

$$
\delta C(x, z)=\sum_{n=1}^{\infty} A_{2 n-1} \cos \left(\frac{(2 n-1) \pi x}{2 S_{\alpha}}\right) \exp \left[\left(-\frac{v}{2 D}-\sqrt{\frac{v^{2}}{4 D^{2}}+\left(\frac{(2 n-1) \pi}{2 S_{\alpha}}\right)^{2}}\right] z\right]
$$

where $A_{2 n-1}$ are constants. Assuming that for slow solidification:

$$
\frac{(2 n-1) \pi}{2 S_{\alpha}}>>\frac{v}{2 D}
$$

Eq. (15) reduces to:

$$
\delta C(x, z)=\sum_{n=1}^{\infty} A_{2 n-1} \cos \left(\frac{(2 n-1) \pi x}{2 S_{\alpha}}\right) \exp \left(-\frac{(2 n-1) \pi}{2 S_{\alpha}} z\right)
$$


The values of $A_{2 n-1}$ parameters are calculated from:

$\left.\frac{\partial \delta C(x, z)}{\partial z}\right|_{z=0}=f_{\alpha}(x) ; \quad f_{\alpha}(x)<0, \quad x \in\left[0, S_{\alpha}\right]$

Applying Eq. (15) or Eq. (15*), the following may be written for rapid and slow solidification:

$$
\begin{aligned}
& \left.\frac{\partial \delta C(x, z)}{\partial z}\right|_{z=0}=\sum_{n=1}^{\infty} A_{2 n-1}\left(-\frac{v}{2 D}-\sqrt{\frac{v^{2}}{4 D^{2}}+\left(\frac{(2 n-1) \pi}{2 S_{\alpha}}\right)^{2}}\right) \cos \left(\frac{(2 n-1) \pi x}{2 S_{\alpha}}\right) \\
& \left.\frac{\partial \delta C(x, z)}{\partial z}\right|_{z=0}=\sum_{n=1}^{\infty} A_{2 n-1}\left(-\frac{(2 n-1) \pi}{2 S_{\alpha}}\right) \cos \left(\frac{(2 n-1) \pi x}{2 S_{\alpha}}\right) .
\end{aligned}
$$

Additionally, it is introduced:

$f(x), \quad-2 S_{\alpha} \leq x \leq 2 S_{\alpha}, \quad f(-x)=f(x), \quad f\left(x+2 S_{\alpha}\right)=-f(x)$.

Next the following property of the $f(x)$ - function is to be applied:

$$
\begin{aligned}
& f(x) \approx \frac{a_{0}}{2}+\sum_{n=1}^{\infty} a_{n} \cos \left(\frac{n \pi x}{2 S_{\alpha}}\right), \\
& a_{n}=\frac{1}{S_{\alpha}} \int_{0}^{2 S_{\alpha}} f(x) \cos \left(\frac{n \pi x}{2 S_{\alpha}}\right) d x .
\end{aligned}
$$

Assuming that $f\left(x+2 S_{\alpha}\right)=-f(x)$ this yields $a_{2 k}=0, k=0,1,2, \ldots$ for $n=2 k$ and $a_{2 k-1}=\frac{2}{S_{\alpha}} \int_{0}^{S_{\alpha}} f(x) \cos \left(\frac{(2 k-1) \pi x}{2 S_{\alpha}}\right) d x, k=1,2, \ldots$ for $n=2 k-1, k=1,2, \ldots$.

Finally, the Fourier series of $f(x)$ is given by

$$
f(x) \approx \sum_{k=1}^{\infty} a_{2 k-1} \cos \left(\frac{(2 k-1) \pi x}{2 S_{\alpha}}\right)
$$

After some rearrangements this can be written: a/ for rapid solidification

$$
A_{2 n-1}=\left(-\frac{v}{2 D}-\sqrt{\frac{v^{2}}{4 D^{2}}+\left(\frac{(2 n-1) \pi}{2 S_{\alpha}}\right)^{2}}\right)^{-1} \frac{2}{S_{\alpha}} \int_{0}^{S_{\alpha}} f_{\alpha}(x) \cos \left(\frac{(2 n-1) \pi x}{2 S_{\alpha}}\right) d x, n=1,2, \ldots
$$

b/ for slow solidification

$$
A_{2 n-1}=-\frac{4}{(2 n-1) \pi} \int_{0}^{S_{\alpha}} f_{\alpha}(x) \cos \left(\frac{(2 n-1) \pi x}{2 S_{\alpha}}\right) d x, \quad n=1,2, \ldots
$$


It can be readily proved that the solution to Eq. (10) given by Eq. (15), Eq. (15*) and Eq. (23), Eq. $\left(23^{*}\right)$ satisfies the conditions:

$$
\begin{aligned}
& \left.\frac{\partial \delta C(x, z)}{\partial x}\right|_{x=0}=\left.\frac{\partial \delta C(x, z)}{\partial x}\right|_{x=2 S_{\alpha}}=0, \\
& \left.\frac{\partial \delta C(x, z)}{\partial z}\right|_{z=0}=f_{\alpha}(x)=f_{\alpha}(-x)=-f_{\alpha}\left(-x+2 S_{\alpha}\right)=-\left.\frac{\partial \delta C\left(-x+2 S_{\alpha}, z\right)}{\partial z}\right|_{z=0}, x \in\left[0, S_{\alpha}\right],
\end{aligned}
$$

according to the assumption: $f_{\alpha}(-x)=f_{\alpha}(x), f_{\alpha}\left(x+2 S_{\alpha}\right)=-f_{\alpha}(x)$.

$\mathrm{B} /$ for the $\beta$ - phase lamella, that is, for $x \in\left[S_{\alpha}, S_{\alpha}+S_{\beta}\right\rfloor, z \geq 0$

The solution of Eq. (10) is given separately for a $\beta$ - phase lamella, however it is found similarly to that for the $\alpha$ - phase lamella. Thus,

a/ for rapid solidification

$$
\begin{aligned}
& \delta C(x, z)=\sum_{n=1}^{\infty} B_{2 n-1} \cos \left(\frac{(2 n-1) \pi\left(x-S_{\alpha}+S_{\beta}\right)}{2 S_{\beta}}\right) \exp \left[\left(-\frac{v}{2 D}-\sqrt{\frac{v^{2}}{4 D^{2}}+\left(\frac{(2 n-1) \pi}{2 S_{\beta}}\right)^{2}}\right] z\right. \\
& B_{2 n-1}=\left(-\frac{v}{2 D}-\sqrt{\frac{v^{2}}{4 D^{2}}+\left(\frac{(2 n+1) \pi}{2 S_{\beta}}\right)^{2}}\right)^{-1} \frac{2}{S_{\beta}} \int_{S_{\alpha}-S_{\beta}}^{S_{\alpha}} f_{\beta}(x) \cos \left(\frac{(2 n+1) \pi\left(x-S_{\alpha}+S_{\beta}\right)}{2 S_{\beta}}\right) d x .
\end{aligned}
$$

b/ for slow solidification

$$
\begin{aligned}
& \delta C(x, z)=\sum_{n=1}^{\infty} B_{2 n-1} \cos \left(\frac{(2 n-1) \pi\left(x-S_{\alpha}+S_{\beta}\right)}{2 S_{\beta}}\right) \exp \left(-\frac{(2 n-1) \pi}{2 S_{\beta}} z\right) \\
& B_{2 n-1}=-\frac{4}{(2 n-1) \pi} \int_{S_{\alpha}-S_{\beta}}^{S_{\alpha}} f_{\beta}(x) \cos \left(\frac{(2 n-1) \pi\left(x-S_{\alpha}+S_{\beta}\right)}{2 S_{\beta}}\right) d x, \quad n=1,2, \ldots
\end{aligned}
$$

where

$$
\left.\frac{\partial \delta C(x, z)}{\partial z}\right|_{z=0}=f_{\beta}(x), \quad x \in\left[0, S_{\beta}\right] .
$$

Both separately obtained solutions of Eq. (10), for slow solidification, for an $\alpha$ - phase lamella, Eq. $\left(15^{*}\right)$ and for a $\beta$ - phase lamella, Eq. $\left(26^{*}\right)$ are shown schematically in Fig. 8a together with the suggested concept of coupled growth, Fig. 8b.

It is evident that both general mass balance and local mass balance should be satisfied within the considered solute concentration micro-field created at the solid/liquid interface for the lamellar eutectic growth. 
The separation of the liquid pre-prepared for solidification is assumed in the current model. Therefore, no adjustment of the volume fractions by the movement of the three-phase junctions are necessary as this was discussed by Magnin and Trivedi [3].

As a simplification, the density difference between eutectic phases is not taken into account when calculating the solute concentration micro-field for lamellar eutectic growth from the separated liquids.

The concept of the coupled growth of eutectic phases ensures the different undercooling of eutectic phases as this was observed experimentally [12].
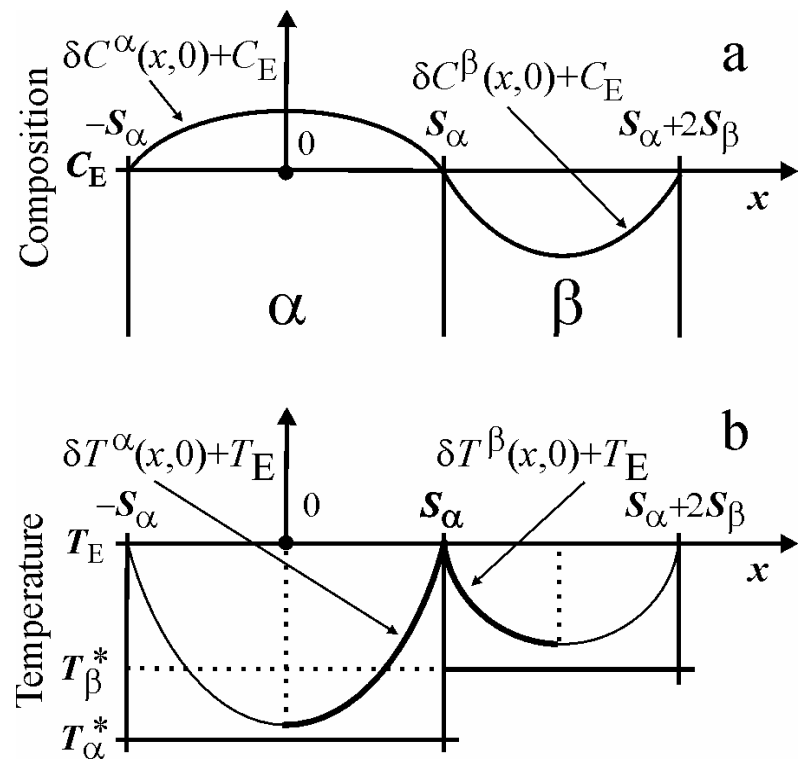

Fig. 8 Calculated: a.) solute profile at $z=0$ and b.) adequate solute and curvature undercooling of the solid / liquid interface due to the current analysis basing on the concept of coupled regular eutectic growth.

\section{Total and local mass balances within the solute concentration micro-field}

A/ At first the general mass balance is analysed in order to find the relationship between $A_{2 n-1}$ and $B_{2 n-1}$ parameters:

$$
\begin{aligned}
& \int_{0}^{\infty} \int_{0}^{S_{\alpha}} \delta C(x, z) d x d z+\int_{0}^{\infty} \int_{S_{\alpha}}^{S_{\alpha}+S_{\beta}} \delta C(x, z) d x d z+=\sum_{n=1}^{\infty} \frac{4(-1)^{n-1} D}{(2 n-1) \pi} \\
& \left(\frac{A_{2 n-1} S_{\alpha}^{2}}{v S_{\alpha}+\sqrt{v^{2} S_{\alpha}^{2}+(2 n-1)^{2} D^{2} \pi^{2}}}-\frac{B_{2 n-1} S_{\beta}^{2}}{v S_{\beta}+\sqrt{v^{2} S_{\beta}^{2}+(2 n-1)^{2} D^{2} \pi^{2}}}\right)=0
\end{aligned} .
$$

a/ for rapid solidification

$$
B_{2 n-1}=\frac{A_{2 n-1} S_{\alpha}^{2}\left(v S_{\beta}+\sqrt{v^{2} S_{\beta}^{2}+(2 n-1)^{2} D^{2} \pi^{2}}\right)}{S_{\beta}^{2}\left(v S_{\alpha}+\sqrt{v^{2} S_{\alpha}^{2}+(2 n-1)^{2} D^{2} \pi^{2}}\right)}, \quad n=1,2, \ldots
$$

b/ for slow solidification

$$
B_{2 n-1}=A_{2 n-1}\left(\frac{S_{\alpha}}{S_{\beta}}\right)^{2}, \quad n=1,2, \ldots
$$


B/ local mass balance

An analysis of the local mass balance proves that it is satisfied at $z=0$ for an $\alpha$ - phase lamella and at $z=d$ for a $\beta$-phase lamella (where $d$ denotes the protrusion of leading phase).

$$
\int_{0}^{S_{\alpha}} \delta C(x, 0) d x+\int_{S_{\alpha}}^{S_{\alpha}+S_{\beta}} \delta C(x, d) d x=0
$$

After some rearrangement:

$$
\sum_{n=1}^{\infty} A_{2 n-1} \frac{2 S_{\alpha}(-1)^{n-1}}{(2 n-1) \pi}-\sum_{n=1}^{\infty} B_{2 n-1} \frac{2 S_{\beta}(-1)^{n-1}}{(2 n-1) \pi} \exp \left(-\frac{v S_{\beta}+\sqrt{v^{2} S_{\beta}^{2}+(2 n-1)^{2} D^{2} \pi^{2}}}{2 D S_{\beta}} d\right)=0 .
$$

Thus, the definition of the $d$ - phase protrusion is:

a/ for rapid solidification

$$
\begin{aligned}
& \sum_{n=1}^{\infty} A_{2 n-1} \frac{(-1)^{n-1}}{(2 n-1)} \times \\
& \left.\left(1-\frac{S_{\alpha}\left(v S_{\beta}+\sqrt{v^{2} S_{\beta}^{2}+(2 n-1)^{2} D^{2} \pi^{2}}\right.}{S_{\beta}\left(v S_{\alpha}+\sqrt{v^{2} S_{\alpha}^{2}+(2 n-1)^{2} D^{2} \pi^{2}}\right.}\right) \exp \left(-\frac{v S_{\beta}+\sqrt{v^{2} S_{\beta}^{2}+(2 n-1)^{2} D^{2} \pi^{2}}}{2 D S_{\beta}} d\right)\right)=0
\end{aligned}
$$

b/ for slow solidification

$$
\sum_{n=1}^{\infty} A_{2 n-1} \frac{(-1)^{n-1}}{(2 n-1)}\left(1-\frac{S_{\alpha}}{S_{\beta}} \exp \left(-\frac{(2 n-1) \pi}{2 S_{\beta}} d\right)\right)=0
$$

$\mathrm{C} / \alpha / \beta$ - boundary mass balance

Taking into account that the local mass balance is satisfied at $z=0$ for the wider $\alpha$ - phase lamella and at $z=d$, for the $\beta$ - phase lamella the boundary mass balance is also considered at the same localization. The existence of the $d$-protrusion has been revealed, Fig. 9a and described theoretically $[13,16]$. It is confirmed experimentally, that usually the minor phase is a leading phase [13]. This corresponds to the analysis of the local mass balance, Eq. (31), Eq. (32), Eq. (33), Eq. $\left(33^{*}\right)$ according to which the $d$ - protrusion is observable at the minor phase interface, only.

A role of the phase protrusion is more visible in the case of irregular eutectics growth as discussed by Chadwick, [16], and Guzik and Burbelko [17].

The s/l interface mass balance is:

$$
S_{\alpha} \frac{\partial \delta C^{\alpha}(x, 0)}{\partial z}=S_{\alpha} \frac{v}{D}\left(1-k_{\alpha}\right) C^{\alpha}(x, 0), \quad x \in\left[0, S_{\alpha}\right]
$$

and 


$$
S_{\beta} \frac{\partial \delta C^{\beta}(x, d)}{\partial z}=S_{\beta} \frac{v}{D}\left(1-k_{\beta}\right) C^{\beta}(x, d), \quad x \in\left[S_{\alpha}, S_{\alpha}+S_{\beta}\right\rfloor
$$

The $\alpha / \beta$ - boundary mass balance is given by the following formula:

$$
\begin{aligned}
& \lim _{x \rightarrow S_{\alpha}^{-}} S_{\alpha} \frac{\partial \delta C^{\alpha}(x, 0)}{\partial z}+\lim _{x \rightarrow S_{\alpha}^{+}} S_{\beta} \frac{\partial \delta C^{\beta}(x, d)}{\partial z}= \\
& S_{\alpha} \frac{v}{D} C_{0}^{\alpha}\left(S_{\alpha}, 0\right)+S_{\beta} \frac{v}{D} C_{0}^{\beta}\left(S_{\alpha}, d\right)=\frac{v}{D}\left(S_{\alpha} C_{0}^{\alpha}\left(S_{\alpha} 0\right)+S_{\beta} C_{0}^{\beta}\left(S_{\alpha}, d\right)\right)=0
\end{aligned} .
$$
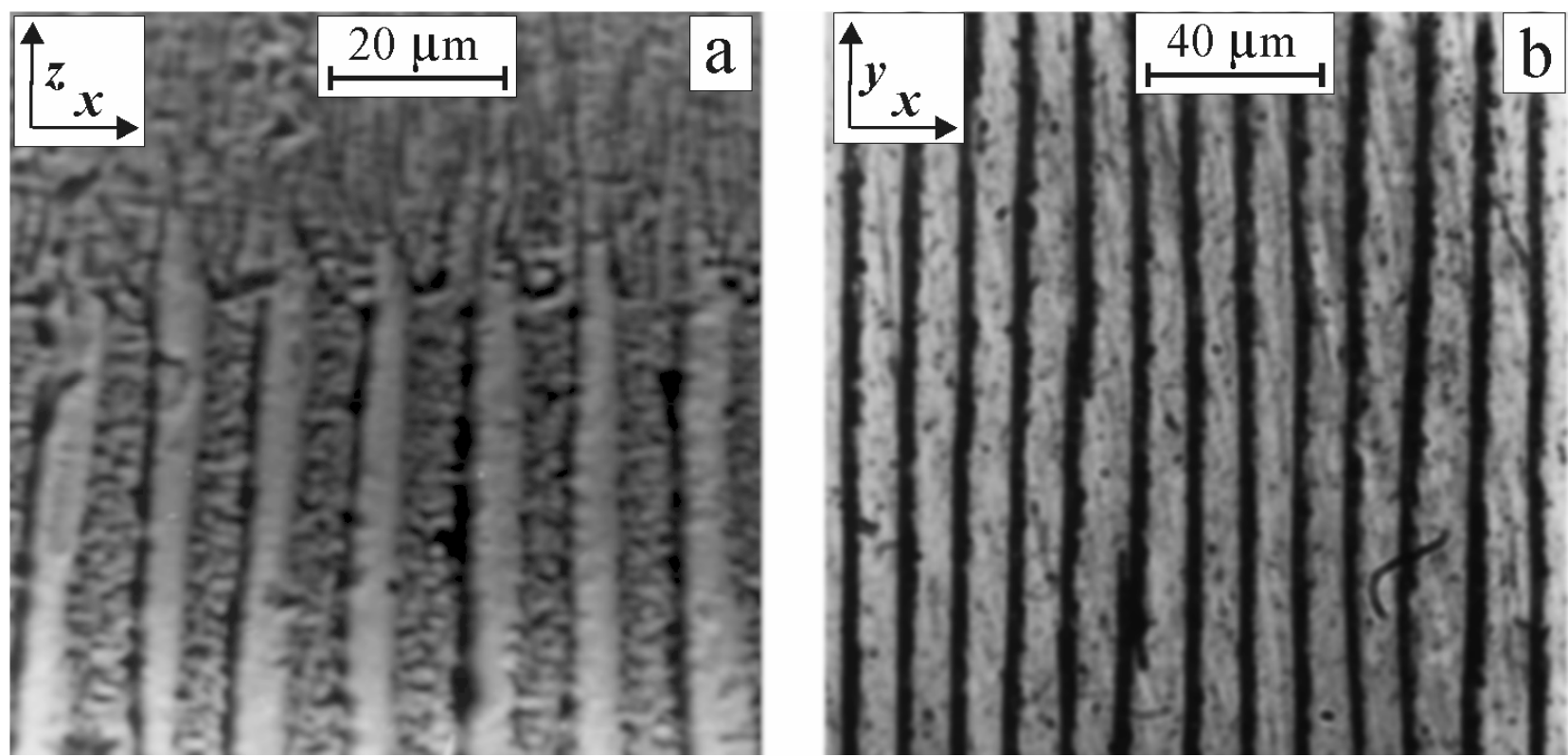

Fig. 9 Morphology of an in situ Pb-Cd composite, as obtained by the Bridgman system, under constant growth rate and constant temperature gradient measured at the solid / liquid interface.

a.) solid / liquid interface frozen during solidification of the $(\mathrm{Pb})-(\mathrm{Cd})$ composite and revealing the protrusion of lamellae of the $(\mathrm{Cd})$ - leading phase; $(\mathrm{Pb})$ - phase is determined as a wetting phase, b.) stationary selection of the lamellae size.

The fulfilment of the $\alpha / \beta$ - boundary mass balance means that thermodynamic equilibrium is ensured at the triple point of the $\mathrm{s} / \mathrm{l}$ interface manifesting the protrusion of the leading phase. Additionally, the above mentioned fulfilment of the mass balance at the $\alpha / \beta$ - boundary justifies an application of the boundary condition formulated by Eq. (14) and illustrated schematically in Fig. 7 and due to the current model calculation in Fig. 8.

The following conditions resulting from the fulfilment of the boundary mass balance are integral parts of both concentration fields shown in Fig. 10 and Fig. 11.

$$
\frac{\partial \delta C\left(S_{\alpha}, z\right)}{\partial z}=\text { const. }, \quad \text { with } \quad C\left(S_{\alpha}, z\right)=C_{E}
$$

This condition, Eq. (37) underlines the existence of assumed separation of both liquids from which some proper composite lamellae are growing. 


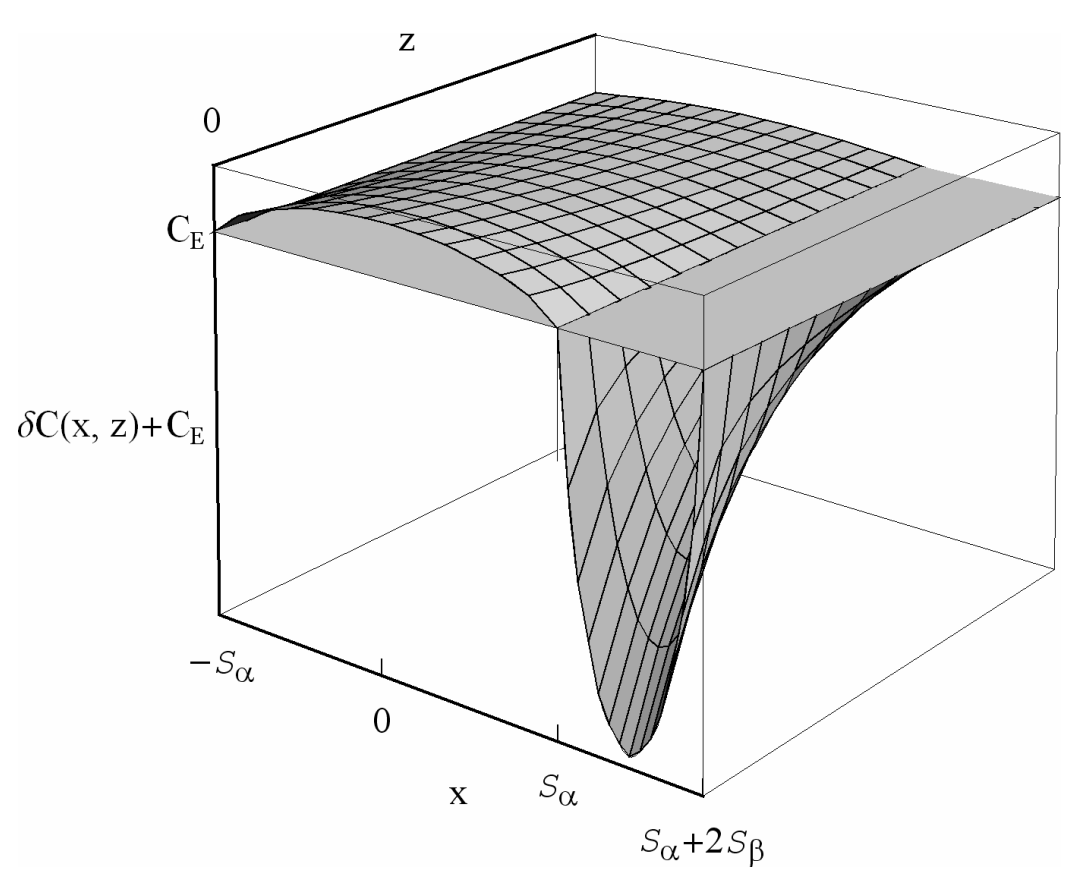

Fig. 10 Micro-field of the solute concentration within the liquid ahead of the solid/liquid interface. The general mass balance within the micro-field is fulfilled.

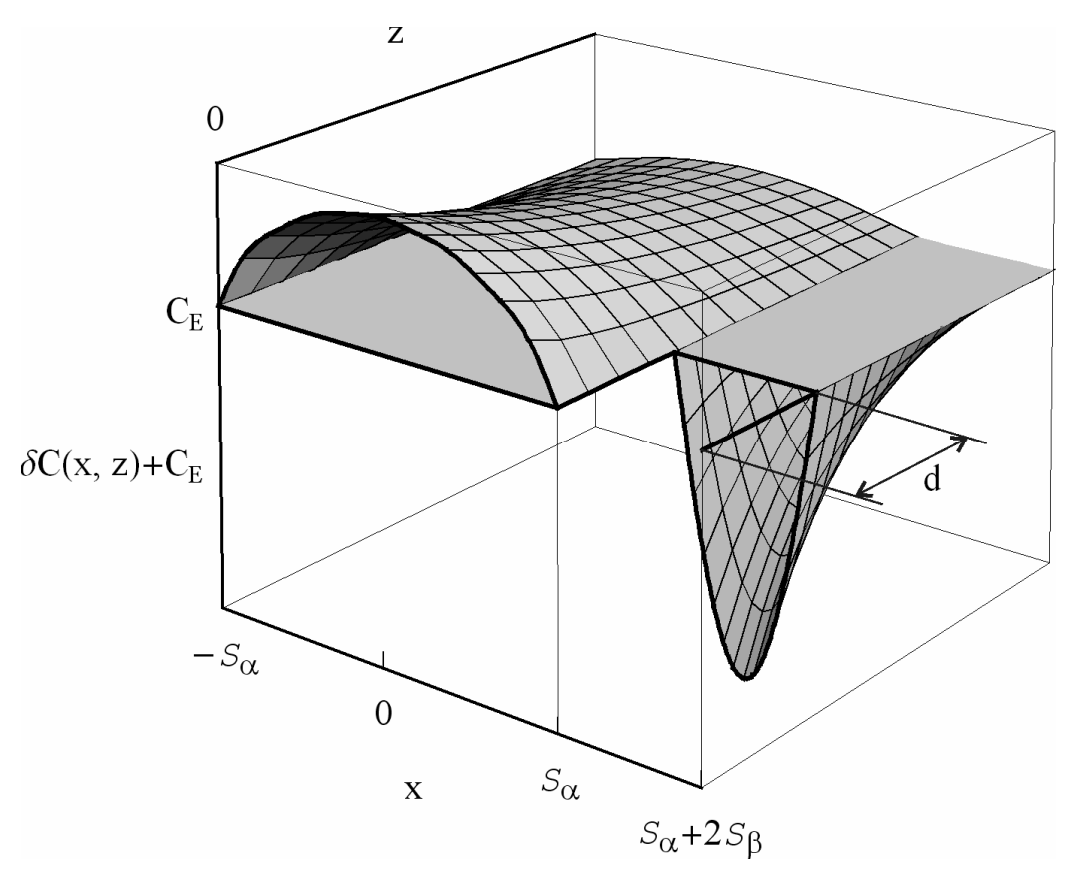

Fig. 11 Micro-field of the solute concentration within the liquid ahead of the $\mathrm{s} / 1$ interface. The $d$ protrusion of the leading phase over the wetting phase is defined. The local mass balance is marked with a bold line encountering solute profile.

\section{Concluding remarks}

The proposed model for coupled growth of both lamellae formed from the separate liquids is favourable for the stable formation of an eutectic structure of a stationary size. Thus, some changes in inter-lamellar spacing cannot be observed, Fig. 9b, when the solute concentration just ahead of the inter-phase boundary is $\left.C\right|_{x=S_{\alpha}}=C_{E}$ for $z \in[0, \infty]$. 
Some perturbations of the separation of both liquids can scarcely lead to unstable growth of the directional structure, Fig. 2a. Therefore, Eq. (14) and Eq. (37) cannot be satisfied in this case.

The $d$ - protrusion is created during eutectic growth as proved by Eq. (33) or Eq. (33*) and confirmed experimentally (Fig. 9a) to ensure the mass balance at the solid / liquid interface of the growing eutectic alloy (see Fig. 11).

Contrary to the concept of ideally coupled growth [2] the current concept of coupled growth introduced in this work is based on the difference in undercooling of the $\mathrm{s} / 1$ interface of both lamellae. The concept of coupled growth is strictly associated with the separation of concentration fields in the liquid and the existence of a protrusion of the leading minor phase to relax the assumption of an ideally isothermal s/l interface.

Equation (16) is satisfied for slow solidification which occurs during in situ composite (oriented eutectic alloy) formation. Therefore, in this case Eq. (30) reduces to Eq. (30*). Moreover it can be proved that for slow solidification during in situ composite growth not only the equation

$B_{2 n-1}=A_{2 n-1}\left(\frac{S_{\alpha}}{S_{\beta}}\right)^{2}, \quad n=1,2, \ldots$

is fulfilled, but

$\left|f_{\beta}\left(x \frac{S_{\beta}}{S_{\alpha}}\right)\right|=\left|f_{\alpha}(x)\right|\left(\frac{S_{\alpha}}{S_{\beta}}\right)^{2}$ as well.

It can also be proved that the current description of the solute concentration micro-fields Eq. (15) or Eq. $\left(15^{*}\right)$ reduces to the description of the solute concentration micro-field given by the J-H theory. It requires, however, to apply some parameters defining the symmetrical phase diagram, for which: $S_{\alpha}=S_{\beta}$. Then, $C_{0}^{\alpha}=C_{0}^{\beta}$, as imposed by the condition $B_{0}=0$, in the J-H theory. In the case of discussed reduction, the protrusion disappears in the present model, $d \rightarrow 0$. It can also be concluded that coupled growth reduces to the so-called ideally coupled growth. As a result the $C_{E}$ solute concentration comes back just to the $\alpha / \beta$ boundary in the $\mathrm{J}-\mathrm{H}$ theory and the local mass balance is satisfied for each $z$ - coordinate (the same coordinate for both lamellae). Consequently in the present model it can be written that $\left|f_{\alpha}\right|=\left|f_{\beta}\right|$. Therefore, the conditions given by Eq. (14) as well as Eq. (37) are also satisfied in the J-H theory. Thus, it is evident that the J-H theory is an excellent approximation only for the so-called symmetrical phase diagrams.

The functions discussed $f_{\alpha}(x)$, required by Eq. (25) and $f_{\beta}(x)$, required by Eq. (27) are schematically illustrated in Fig. 12. The present model presents some relationships not only for in situ composite growth (slow solidification) but is developed for rapid solidification as well, when inequality given by Eq. (16) is not satisfied and the term, $\frac{v}{2 D}$, cannot be neglected. 

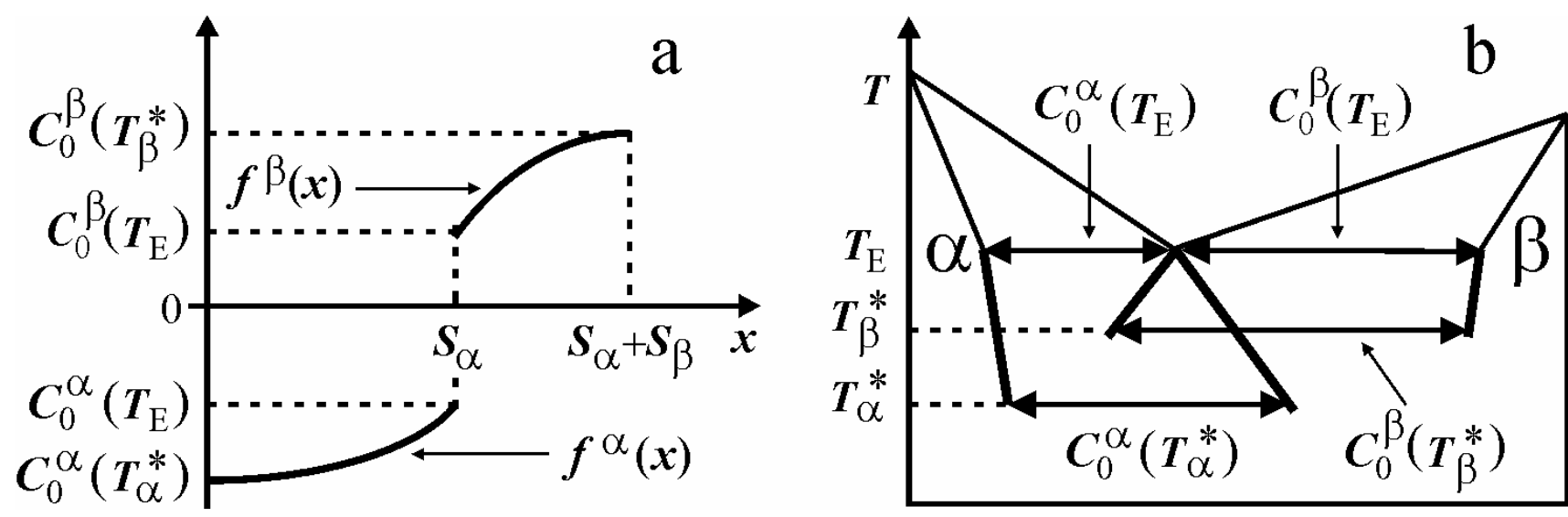

Fig. 12 Schematic presentation of the $f^{\alpha}(x)$ and $f^{\beta}(x)$ functions: a.) limitation of the discussed functions resulting from the concept of coupled growth, b.) the concept of coupled growth related to an arbitrary phase diagram.

The use of the concept of coupled growth, Fig. 12b, and the new boundary condition, Eq. (9), allowed to deliver the solution to diffusion equation according to which the separation plane within the liquid exists. The thermodynamic equilibrium is located onto this plane.

The location of thermodynamic equilibrium is important while considering an entropy production for the thermo-diffusion phenomena occurring during stationary formation of the lamellar eutectic structure. The envisaged equilibrium should be contained within the so-called macroscopic point (volume) required by the calculation of entropy production per unite time as suggested by Glansdorff and Prigogine [18] and discussed by Lesoult and Turpin [19] as well as by Wołczyński and Billia [20]. Usually, the thermodynamic equilibrium was attributed to the solid / liquid interface [19], [20].

According to the assumptions used in the model based on the concept of coupled growth the thermodynamic equilibrium is to be attributed to the separation plane situated within the liquid. The separation plane makes a lengthening of the $\alpha / \beta$ boundary existing between solid phases.

\section{Acknowledgements}

The work was financially supported by the Polish Ministry of Science and Higher Education, Project No 3T08C 00327.

The author thanks Professor Edward Fraś with the Faculty of Foundry Engineering, AGH University of Science and Technology in Kraków, Poland for remarks and fruitful discussion and Professor Signe Kjelstrup with the Department of Chemistry, Faculty of Natural Science and Technology, Norwegian University of Science and Technology, Trondheim, Norway for some comments. 


\section{Symbols used in the text}

$C \quad$ - solute concentration within the liquid,

$C_{E} \quad$ - equilibrium eutectic concentration of solute,

$C^{\alpha} \quad$ - solute concentration in the liquid at the solid / liquid interface ahead of the $\alpha$ - phase lamella,

$C^{\beta} \quad$ - solute concentration in the liquid at the solid / liquid interface ahead of the $\beta$ - phase lamella,

$C_{S}^{\alpha} \quad$ - equilibrium solute concentration in the $\alpha$ - phase lamella,

$C_{S}^{\beta} \quad$ - equilibrium solute concentration in the $\beta$ - phase lamella,

$d \quad$ - protrusion of the leading minor phase, $\beta$, and concentration field parameter,

$D \quad$ - diffusion coefficient in the liquid,

$f_{\alpha} \quad$ - function used in formulation of boundary condition for the $\alpha$ - phase,

$f_{\beta} \quad$ - function used in formulation of boundary condition for the $\beta$ - phase,

$k_{\alpha} \quad$ - partition ratio for the formation of the $\alpha$ - phase lamella,

$k_{\beta} \quad$ - partition ratio for the formation of the $\beta$ - phase lamella,

$S_{\alpha} \quad$ - half the width of the $\alpha$ - phase lamella,

$S_{\beta} \quad$ - half the width of the $\beta$ - phase lamella,

$T \quad$ - temperature,

$T^{\alpha} \quad$ - interface temperature resulting from the concentration field for the $\alpha$ - phase,

$T^{\beta} \quad$ - interface temperature resulting from the concentration field for the $\beta$ - phase,

$T_{\alpha}^{*} \quad$ - real temperature of the s/l interface of the $\alpha$ - phase,

$T_{\beta}^{*} \quad$ - real temperature of the s/l interface of the $\beta$ - phase,

$T_{E} \quad$ - equilibrium eutectic temperature,

$v \quad$ - stationary growth rate of the composite in situ,

$x \quad$ - co-ordinate system axis parallel to the solid / liquid interface, for the co-ordinate system attached to the interface,

$z \quad$ - co-ordinate system axis perpendicular to the solid / liquid interface, for the co-ordinate system attached to the interface,

$\alpha \quad$ - wetting eutectic phase, (lamella within the composite in situ),

$\beta \quad$ - leading eutectic phase, (lamella within the composite in situ),

$\delta C^{\alpha} \quad$ - concentration field in the liquid ahead the s/l interface of the $\alpha$ - phase,

$\delta C^{\beta} \quad$ - concentration field in the liquid ahead the s/l interface of the $\beta$ - phase,

$\Delta T_{\alpha}^{*} \quad$ - total undercooling of the s/l interface of the $\alpha$ - phase,

$\Delta T_{\beta}^{*} \quad$ - total undercooling of the $\mathrm{s} / 1$ interface of the $\beta$ - phase,

$\delta T^{\alpha} \quad$ - solute undercooling of the s/l interface of the $\alpha$ - phase,

$\delta T^{\beta} \quad$ - solute undercooling of the s/l interface of the $\beta$ - phase,

$\delta T_{R}^{\alpha} \quad$ - curvature undercooling of the s/l interface of the $\alpha$ - phase,

$\delta T_{R}^{\beta} \quad$ - curvature undercooling of the s/l interface of the $\beta$-phase. 


\section{References}

[1] K.A.Jackson and J.D.Hunt: Trans. AIME Vol. 236, (1966), p. 1129

[2] J.D.Hunt and K.A.Jackson: Trans. AIME Vol. 236, (1966), p. 843

[3] P.Magnin and R.Trivedi: Acta Metall. Mater. Vol. 39, (1991), p. 453

[4] H.E.Cline: Trans. AIME Vol. 242, (1968), p. 1613

[5] D.T.Hurle and E.Jakeman: J. of Crystal Growth Vol. 3-4, (1968), p. 574

[6] S.Strassler and W.R.Schneider: Phys. Condens. Matter Vol. 17, (1974), p. 153

[7] V.Datye and J.S.Langer: Phys. Rev. Vol. 24B, (1981), p. 4155

[8] A.Karma and A.Sarkissian: J. of Comp. Phys. Vol. 17, (1995), p. 143

[9] R.Elliott, in: Growth in Normal Eutectic Solidification, Chapter 3 of Eutectic Solidification Processing, Butterworths Monographs in Materials, Bodmin, Cornwall (1983)

[10] J.W.Rutter: J. of Crystal Growth Vol. 42, (1977), p. 515

[11] W.Kurz and D.J.Fisher, in: Solidification Microstructure: Eutectic and Peritectic, Chapter 5 of Fundamentals of Solidification, Trans Tech Publications, Switzerland (1992)

[12] P.B.Crosley, A.W.Douglas and L.F.Mondolfo, in: Interfacial Energies in Heterogeneous Nucleation, Chapter 2 of The Solidification of Metals, The Iron and Steel Institute, London, (1968).

[13] W.Wołczyński: Crystal Research and Technology Vol. 25, (1990), p. 1303

[14] W.Wołczyński: Crystal Research and Technology Vol. 27, (1992), p. 195

[15] V.L.Davies: Journal of the Institute of Metals Vol. 93, (1964/65), p. 10

[16] G.A.Chadwick, in: Eutectic Alloy Solidification, Chapter 2 in: Progress in Materials Science, Pergamon Press, Headington Hill Hall, Oxford, (1964)

[17] E.Guzik and A.Burbelko, Proceedings of the 4th International Conference "Eutectica IV", Dniepropietrovsk, (1997), p. 67

[18] P.Glansdorff and I.Prigogine, Physica Vol. 30, (1964), p.351.

[19] G.Lesoult and M.Turpin, Memoires Scientifiques de la Revue de Metallurgie Vol. 66, (1969), p. 619

[20] W.Wołczyński and B.Billia, Mat. Science Forum Vol. 215/216, (1996), p. 313. 